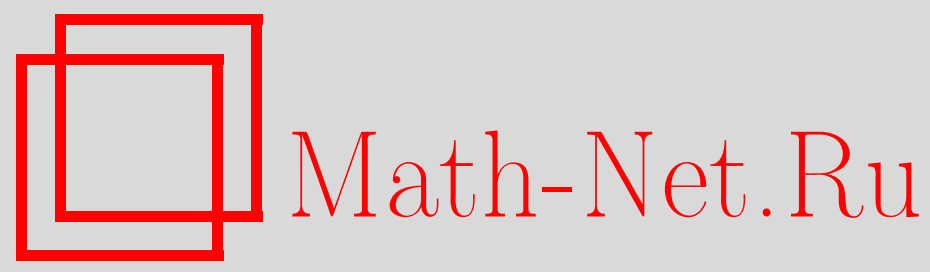

Л. Ю. Чередникова, Последовательности неединственности для весовых алгебр голоморфных функций в единичном круге, Матем. заметки, 2005, том 77, выпуск 5, 775-787

DOI: https://doi.org/10.4213/mzm2523

Использование Общероссийского математического портала Math-Net.Ru подразумевает, что вы прочитали и согласны с пользовательским соглашением http://www.mathnet.ru/rus/agreement

Параметры загрузки:

IP: 18.209 .158 .208

26 апреля 2023 г., 18:03:28

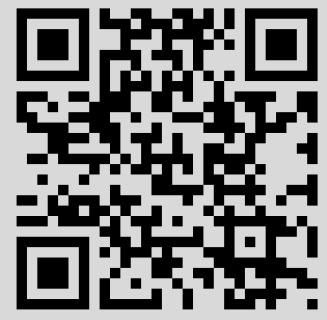




\section{ПОСЛЕДОВАТЕЛЬНОСТИ НЕЕДИНСТВЕННОСТИ ДЛЯ ВЕСОВЫХ АЛГЕБР ГОЛОМОРФНЫХ ФУНКЦИЙ В ЕДИНИЧНОМ КРУГЕ}

\section{Л. Ю. Чередникова}

Пусть $\mathscr{P}$ - система непрерывных субгармонических функций в единичном круге $\mathbb{D}$ и $A_{\mathscr{P}}$-класс голоморфных в $\mathbb{D}$ функций $f$ таких, что $\log |f(z)| \leqslant B_{f} p_{f}(z)+C_{f}, z \in \mathbb{D}$, где $B_{f}$ и $C_{f}$ - постоянные, а $p_{f} \in \mathscr{P}$. Получены достаточные условия, при которых заданная последовательность чисел $\Lambda=\left\{\lambda_{n}\right\} \subset \mathbb{D}$ является подпоследовательностью нулей какой-нибудь ненулевой голоморфной функции из $A \mathscr{P}$, т.е. $\Lambda$ - последовательность неединственности для $A \mathscr{P}$.

Библиографоия: 17 названий.

Введение. Круг известных описаний нулевых последовательностей и последовательностей неединственности для различных алгебр голоморфных функций в единичном круге $\mathbb{D}=\{z \in \mathbb{C}:|z|<1\}$ комплексной плоскости $\mathbb{C}$ очень обширен. Не претендуя на полноту охвата библиографии по этой тематике, отметим здесь лишь обзор С. В. Шведенко [1], монографию А. Джрбашяна и $\Phi$. А. Шамояна [2] и наиболее близкие к настоящей работе по типу рассматриваемых алгебр результаты законченного характера Шамояна [3], [4], продолжившего исследования М. М. Джрбашяна, а также статьи Ч. Горовица, Б. Коренблюма, И. Беллера, И. Бруно и К. Массанеда [5]-[9]. При этом весовые функции $p$, определяющие алгебру голоморфньх функций посредством оценок вида $|f| \leqslant C_{f} \exp \left(c_{f} p\right), C_{f}, c_{f}$ - постоянные, рассматривались, как правило, радиальные, т.е. зависящие только от расстояния до нуля, и положительные. В настоящей работе приводятся достаточные условия, при которых заданная последовательность $\Lambda=\left\{\lambda_{n}\right\} \subset \mathbb{D}$-последовательность неединственности для алгебры голоморфных в круге функций в случае, когда весовые функции, определяющие алгебру, лишь субгармоничны и непрерьвны, но, вообще говоря, не радиальны и могут стремиться к $-\infty$ при приближении к отдельным участкам границы (теорема неединственности). В то же время теорема неединственности является новой и для радиальных и положительных весовых функций (следствие). Как показывает сравнение с известньци результатами Шамояна [3, теорема 2.2], [4, теорема А] о полном описании нулевых множеств для некоторых алгебр с радиальными весами, теорема неединственности и ее следствие точны во всяком случае для алгебр, определяемых умеренно растущими радиальными весами.

Работа выполнена при поддержке Российского фонда фундаментальных исследований, грант № 03-01-00033. 
Автор выражает глубокую признательность Б.Н. Хабибуллину, под чьим руководством вьполнена эта работа.

1. Основные результаты. Всюду положительность числа, функции, меры и т.п. понимаем как $\geqslant 0, a>0$ суть строгая положительность; аналогичное соглашение предлагается и для отрицательности.

Пусть $\Lambda=\left\{\lambda_{n}\right\}$ - последовательность точек в $\mathbb{D}$ и $\Lambda$ не имеет предельных точек в $\mathbb{D}$; $H$ - некоторое векторноепространство надполем $\mathbb{C}$ голоморфных в $\mathbb{D}$ функций. Последовательность $\Lambda$ называется последовательностью неединственности для пространства $H$, если существует ненулевая функция $f \in H$, обращающаяся в нуль на $\Lambda$ в том смысле, что кратность нуля функции $f$ в каждой точке из $\mathbb{D}$ не меньше числа повторений этой точки в последовательности $\Lambda$ (пишем $f(\Lambda)=0)$.

Рассматривается следующий тип весовых алгебр.

Пусть $\mathscr{P}$ - система непрерьвных субгармонических функций в $\mathbb{D}$. Если система $\mathscr{P}$ обладает свойством

(A) для любих $p_{1} \in \mathscr{P} u p_{2} \in \mathscr{P} u$ для любых постоянных $c_{1}, c_{2}>0$ найдутся функиия $p_{3} \in \mathscr{P}$ и постоянные $C_{1}, C_{2}>0$ такие, что

$$
\max \left\{c_{1} p_{1}(z), c_{2} p_{2}(z)\right\} \leqslant C_{1} p_{3}(z)+C_{2}, \quad z \in \mathbb{D},
$$

то класс голоморфных в $\mathbb{D}$ функций $f$, удовлетворяющих оценке

$$
\log |f(z)| \leqslant A_{f} p_{f}(z)+B_{f}, \quad z \in \mathbb{D},
$$

где $A_{f}$ и $B_{f}$ - строго положительные постоянные, а $p_{f}$ - некоторая функция из $\mathscr{P}$, образует алгебру, которую обозначаем далее через $A_{\mathscr{P}}$. Действительно, если $f, g \in A_{\mathscr{P}}$, т.е. вместе с (1.2) справедлива оценка

$$
\log |g(z)| \leqslant A_{g} p_{g}(z)+B_{g}, \quad z \in \mathbb{D}
$$

где постоянные $A_{g}, B_{g}>0$, то по условию $(\mathbf{A})$, примененному к функциям $p_{1}=p_{f}$ и $p_{2}=p_{g}$ и постоянньм $c_{1}=A_{f}$ и $c_{2}=A_{g}$, получаем оценки

$$
\begin{aligned}
\log |f+g| & \leqslant \log (|f|+|g|) \leqslant \max \{\log |f|, \log |g|\}+\log 2 \\
& \leqslant \max \left\{A_{f} p_{f}+B_{f}, A_{g} p_{g}+B_{g}\right\}+\log 2 \\
& \leqslant \max \left\{A_{f} p_{f}, A_{g} p_{g}\right\}+\max \left\{B_{f}, B_{g}\right\}+\log 2 \leqslant C_{1} p+C_{2}
\end{aligned}
$$

для некоторой функции $p \in \mathscr{P}$ и постоянных $C_{1}, C_{2}>0$, т.е. класс $A \mathscr{P}$ замкнут относительно сложения. Очевидно, $\alpha f \in A_{\mathscr{P}}$ для любого $\alpha \in \mathbb{C}$. Наконец, при условии $(\mathbf{A})$

$$
\begin{aligned}
\log |f g| & \leqslant 2 \max \{\log |f|, \log |g|\} \leqslant 2 \max \left\{A_{f} p_{f}+B_{f}, A_{g} p_{g}+B_{g}\right\} \\
& \leqslant 2 \max \left\{A_{f} p_{f}, A_{g} p_{g}\right\}+2 \max \left\{B_{f}, B_{g}\right\} \leqslant C_{1} p+C_{2}
\end{aligned}
$$

для некоторой функции $p \in \mathscr{P}$ и постоянных $C_{1}, C_{2}>0$, т.е. класс $A \mathscr{P}$ замкнут и относительно умножения. Отметим, что если все функции из класса $\mathscr{P}$ положительны, то выполнение условия $(\mathbf{A})$ достаточно проверить при $c_{1}=c_{2}=1$. 
Для подмножества $D \subset \mathbb{C}$ через $\partial D, \operatorname{diam} D$ и $\operatorname{dist}(z, D)$ обозначаем соответственно гранищу множества $D$, диаметр $D$ и евклидово расстояние от точки $z \in \mathbb{C}$ до $D$. Через $D(z, t)$ обозначаем открытьй круг с центром в точке $z \in \mathbb{C}$ радиуса $t ; D(t) \stackrel{\text { def }}{=} D(0, t)$.

Если $\nu$ - мера и $\Lambda$ - последовательность точек в $\mathbb{D}$, то $\nu(D)$ и $\Lambda(D)$ - соответственно $\nu$-мера множества $D$ и число точек последовательности $\Lambda$, попавших в $D$.

Для субгармонической функции $p$ через $\nu_{p}$ обозначаем распределение масс Рисса (ассоциированную меру) функции $p$.

Для функции $R(t)$, определенной на интервале $[0,1)$, полагаем

$$
R_{\beta}(t) \stackrel{\text { def }}{=} R\left(\frac{(t-\beta)^{+}}{1-\beta}\right), \quad 0 \leqslant \beta<1, \quad t \in[0,1),
$$

где $a^{+} \stackrel{\text { def }}{=} \max \{a, 0\}$. Основной результат статьи, анонсированный в несколько более слабой форме в [10], следующий.

ТЕОРЕМА НЕЕДИНСТВЕННОСТИ. Пусть Я - система непрерывных субгармонических функций в $\mathbb{D}$, удовлетворяющих условию $(\mathbf{A}), R(t)$ - убьвающая строго положстельная непрерывная функиия на $[0,1)$ такая, что для некоторого числа $\beta \leqslant 1 / 2$

$$
\begin{aligned}
& R(t) \leqslant \beta(1-t) \quad \text { npu } \quad \beta<\frac{1}{2}, \quad 0 \leqslant t<1, \\
& R(t)<\frac{1}{2}(1-t) \quad \text { npu } \quad \beta=\frac{1}{2}, \quad 0 \leqslant t<1 \text {. }
\end{aligned}
$$

Если для системы ЯР выполнены два условия:

(R) для любой функиии $p \in \mathscr{P}$ найдутся функиия $p_{1} \in \mathscr{P}$ и постоянные $C_{1}, C_{2}>0$ такие, что при некотором $t_{0}<1$

$$
\frac{1}{2 \pi} \int_{0}^{2 \pi} p\left(z+R_{\beta}(|z|) e^{i \theta}\right) d \theta \leqslant C_{1} p_{1}(z)+C_{2}, \quad t_{0} \leqslant|z|<1
$$

(S) для любой функиии $p_{1} \in \mathscr{P}$ и постоянной $C>0$ найдутся функиия $p_{2} \in \mathscr{P}$ $u$ постоянные $C_{1}, C_{2}>0$ такие, что при некотором $t_{0}<1$ в каждой точке $z,|z| \geqslant t_{0}$, для некоторого числа $\sigma$ (зависящего от $z$ ), $0<\sigma<1-|z|$, выполнена оченка

$$
\sup _{|\zeta-z|<\sigma} p_{1}(\zeta)+C \log \frac{1}{\sigma} \leqslant C_{1} p_{2}(z)+C_{2}, \quad t_{0} \leqslant|z|<1
$$

и при этом для некоторой системы $\left\{D_{k}\right\}, k=1,2, \ldots$, непересекаюшихся борелевских множсетв выполнены соотношения

$$
\limsup _{k \rightarrow \infty} \frac{\operatorname{diam} D_{k}}{R\left(d_{k}\right)}<1, \quad 1>d_{k} \stackrel{\text { def }}{=} \sup \left\{|z|: z \in D_{k}\right\} \underset{k \rightarrow \infty}{\longrightarrow} 1
$$


а также последовательность $\Lambda \subset \bigcup_{k} D_{k}$ для некоторой функиии $p \in \mathscr{P}$ удовлетворяет условию

$$
\limsup _{k \rightarrow \infty} \frac{\Lambda\left(D_{k}\right)}{\nu_{p}\left(D_{k}\right)}<\infty
$$

әде $\nu_{p}$ - распределение масс Рисса функиии $p$, то $\Lambda$ - последовательность неединственности для $A_{p}$.

Выполнение условий $(\mathbf{R})$ и $(\mathbf{S})$ требуется лишь при $t$, близких к 1 . Поэтому в теореме неединственности, вообще говоря, достаточно считать убывающую строго положительную функцию $R(t)$ определенной лишь на некотором интервале $\left[t^{\prime}, 1\right), 0 \leqslant t^{\prime}<1$. В этом случае можно продолжить ее на весь интервал $[0,1)$ с сохранением условия (1.4), полагая $R(t) \equiv R\left(t^{\prime}\right)$ при $0 \leqslant t \leqslant t^{\prime}$. Тогда в силу непрерьвности функций из $\mathscr{P}$, увеличивая, если это необходимо, постоянную $C_{2}>0$ в (1.5) и $(1.6)$, условия $(\mathbf{R})$ и $(\mathbf{S})$ также можно считать вьполненными при всех $z \in \mathbb{D}$ с продолженной на весь интервал $[0,1)$ функцией $R(t)$. Условия (1.7) и (1.8) также сохраняются ввиду их асимптотического характера.

Отметим также, что условие (1.4) обеспечивает (см. лемму 2 , оценку (4.14)) то, что $z+R_{\beta}(|z|) e^{i \theta} \in \mathbb{D}$ для всех $z \in \mathbb{D}$, т.е. левая часть в (1.5) определена при всех $z \in \mathbb{D}$.

Доказательство теоремы неединственности следует схеме доказательства аналогичных результатов для алгебр и пространств целых функций из [11, теорема 3.1$]$ (см. также элементы этой схемы в [12]). В отличие от традиционных методов описания нулевых последовательностей и последовательностей неединственности из [1]-[9] это доказательство неконструктивно в том смысле, что не использует каких-либо конкретных представлений голоморфных функций с заданными ограничениями на рост вблизи гранишы $\partial \mathbb{D}$.

Несколько ослабляя теорему неединственности, два условия $(\mathbf{R})$ и $(\mathbf{S})$ можно объединить в одно.

СлЕдСТВИЕ. Пусть функиия $R(t)$ такая же, как в теореме неединственности. Если система Я непрерывных субгармонических положительных функиий удовлетворяет условию

(RS) для любой функиии $p \in \mathscr{P}$ и для любой постоянной $С$ найдутся функиия $p_{1} \in \mathscr{P}$ и постоянные $C_{1}, C_{2}>0$ такие, что при некотором $t_{0}<1$ выполнены неравенства

$$
\sup _{|z-\zeta| \leqslant R_{\beta}(|z|)} p(\zeta)+C \log \frac{1}{R_{\beta}(|z|)} \leqslant C_{1} p_{2}(z)+C_{2}, \quad t_{0} \leqslant|z|<1
$$

и при этом для некоторой системы $\left\{D_{k}\right\}, k=1,2, \ldots$, непересекающихся борелевских множсеств выполнены соотношения (1.7) и (1.8), то $\Lambda$ - последовательность неединственности для $A_{p}$.

Действительно, условие $(\mathbf{R S})$ влечет за собой свойство $(\mathbf{R})$ по принципу максимума для субгармонических функций и свойство $(\mathbf{S})$ для значений $\sigma=\sigma(z)=R_{\beta}(|z|)$. 
2. Применение мер и функций Йенсена. Основные свойства мер Йенсена, их потенциалов и функций Йенсена можно найти в [13].

Как обычно, $\operatorname{supp} \nu-$ носитель меры $\nu$.

Положительную борелевскую меру $\mu$ с компактным носителем $\operatorname{supp} \mu$ в $\mathbb{D}$ назьвают мерой Йенсена (для круга $\mathbb{D})$, если для любой субгармонической в $\mathbb{D}$ функции $u$ вьполнено неравенство $u(0) \leqslant \int u d \mu$. Мера Йенсена есть ни что иное, как выметание меры Дирака, т.е. единичной массы, сосредоточенной в нуле.

Субгармоническую в $\mathbb{C} \backslash\{0\}$ функцию $V$ назьваем функиией Йенсена (для круга $\mathbb{D})$, если функция $V$ неотрицательна, тождественно равна нулю вне некоторого круга радиуса менее 1 с центром в нуле и справедливо асимптотическое соотношение $V(\zeta) \leqslant$ $-\log |\zeta|+O(1)$ при $\zeta \rightarrow 0$.

Если $\mu$-мера Йенсена, то функция

$$
V_{\mu}(\zeta)=\int \log |z-\zeta| d \mu(z)-\log |\zeta|
$$

назьваемая далее потенииалом меры $\mu$, является функцией Йенсена. Соответствие $\mu \rightarrow V_{\mu}$ задает биекцию класса всех мер Йенсена для $\mathbb{D}$ на класс всех функций Йенсена для $\mathbb{D}$.

Имеет место следующее обобщение классической формулы Пуассона-Йенсена: $е с л и$ $p$ - субгармоническая функиия в $\mathbb{D}$ с распределением масс Рисса $\nu_{p}, p(0) \neq-\infty, u$ $\mu$ - мера Йенсена для $\mathbb{D}$, то выполнено равенство

$$
\int p d \mu=\int V_{\mu} d \nu_{p}+p(0) .
$$

Если функция $f$ не равна тождественно величине $a \in[-\infty,+\infty]$, то пишем $f \not \equiv a$.

В основу нашего исследования положен общий результат Хабибуллина из [14, теорема 1] (различные вариации на ту же тему см. в его же работах [13, основная теорема], $[15$, теорема 1.3], [16, §11], а также в книге П. Кусиса [12, гл. III, § С.1, теорема Хабибуллина]), которьй мы формулируем здесь частично и лишь для круга $\mathbb{D}$.

ПРЕДЛОЖЕНИЕ 1 [14, теорема 1]. Пусть и-субгармоническая функиия в $\mathbb{D}, M-$ непрерывная вещественнозначная функиия в $\mathbb{D}$. Если существует постоянная $C$ такая, что для всех мер Йенсена $\mu$ (для круга $\mathbb{D})$ выполнено неравенство

$$
\int u d \mu \leqslant \int M d \mu+C
$$

то найдется голоморфная в $\mathbb{D}$ функиия $h \not \equiv 0$ такая, что для любой точки $z \in \mathbb{D}$ и любого $\sigma, 0<\sigma<\operatorname{dist}(z, \partial \mathbb{D})$, выполнено неравенство

$$
u(z)+\log |h(z)| \leqslant \sup _{|z-\zeta| \leqslant \sigma} M(\zeta)+3 \log (1+|z|+\sigma)-\log \sigma .
$$

Придадим этому предложению удобную для применения в настоящей работе форму (ср. [13, основная теорема]). 
Последовательность $\Lambda$ называется последовательностью нулей голоморфной в $\mathbb{D}$ функции $f$, если кратность нуля функции $f$ в каждой точке из $\mathbb{D}$ совпадает с числом повторений этой точки в последовательности $\Lambda$. Если $\Lambda$ - последовательность нулей функции $f$, то часто удобно отождествлять эту последовательность с мерой, обозначаемой тем же символом $\Lambda$, такой, что $\Lambda(B)$ - число точек последовательности $\Lambda$, попавших в $B \subset \mathbb{D}$. Связано это с тем, что распределение масс Рисса субгармонической функции $\log |f|$ есть мера $\Lambda$.

ПРЕДЛОЖЕНИЕ 2. Пусть $\Lambda=\left\{\lambda_{n}\right\}$ - последовательность точек в $\mathbb{D}$, не имеющая предельных точек в $\mathbb{D}, 0 \notin \Lambda, u M$ - непрерывная вещественнозначная функиия в $\mathbb{D}$. Если существует постоянная $C$ такая, что для всех мер Йенсена $\mu$ выполнено неравенство

$$
\sum_{n} V_{\mu}\left(\lambda_{n}\right) \leqslant \int M d \mu+C
$$

то найдется голоморфная в $\mathbb{D}$ функиия $f \not \equiv 0$ такая, что $f(\Lambda)=0$ и для любой строго положительной функции $\sigma(z)<1-|z|$ выполнена оценка

$$
\log |f(z)| \leqslant \sup _{|z-\zeta| \leqslant \sigma(z)} M(\zeta)+\log \frac{1}{\sigma(z)} .
$$

ДоКАЗАТЕЛЬСТВо. Из начальных условий на последовательность $\Lambda$ по классической теореме Вейерштрасса следует, что сушествует голоморфная в $\mathbb{D}$ функция $f_{\Lambda}$ с последовательностью нулей в точности $\Lambda, f_{\Lambda} \neq 0$. Применяя обобщенную формулу Пуассона-Йенсена (2.1) к субгармонической функции $p=\log \left|f_{\Lambda}\right|$ с распределением масс Рисса $\Lambda$, получаем

$$
\int \log \left|f_{\Lambda}(z)\right| d \mu(z)=\int V_{\mu} d \Lambda+\log \left|f_{\Lambda}(0)\right|=\sum_{n} V_{\mu}\left(\lambda_{n}\right)+\log \left|f_{\Lambda}(0)\right| .
$$

Из этого равенства и условия (2.4) вытекает существование постоянной $C$ такой, что неравенство

$$
\int \log \left|f_{\Lambda}(z)\right| d \mu(z) \leqslant \int M d \mu+C+\log \left|f_{\Lambda}(0)\right|
$$

выполнено для всех мер Йенсена $\mu$. Отсюда по предложению 1 с постоянной $C^{\prime}=C+$ $\log \left|f_{\Lambda}\right|(0)$ вместо $C$ найдется голоморфная в круге $\mathbb{D}$ функция $h \not \equiv 0$ такая, что для любой точки $z \in \mathbb{D}$ и для любой строго положительной функции $\sigma(z)<1-|z|$ (при выборе $\sigma=\sigma(z))$ вьполнена оценка

$$
\begin{aligned}
\log \left|f_{\Lambda}(z)\right|+\log |h(z)| & \leqslant \sup _{|z-\zeta| \leqslant \sigma} M(\zeta)+3 \log (1+|z|+\sigma(z))-\log \sigma \\
& \leqslant \sup _{|z-\zeta| \leqslant \sigma} M(\zeta)+3 \log 2+\log \frac{1}{\sigma} .
\end{aligned}
$$

Последнее при $f=2^{-3} f_{\Lambda} h$ дает в точности (2.5). При этом по построению $f \not \equiv 0$ и $f(\Lambda)=0$. 
3. Лемма сравнения. Доказательство следующей леммы, играющей ключевую роль, следует доказательству результата из [11, лемма 1.1] (см. также [12, гл. III, C, лемма сравнения]). Полагаем $\log ^{+} t=\max \{0, \log t\}$.

ЛЕмМА СРАВНЕНИЯ. Пусть $\lambda u \nu$ - две положительные борелевские меры $c$ носителями в $\mathbb{D}$, представленные в виде суммы положительных мер с компактными носителями

$$
\lambda=\sum_{k=1}^{\infty} \lambda^{(k)}, \quad \nu=\sum_{k=1}^{\infty} \nu^{(k)}
$$

mak, чmo

$$
\lambda^{(k)}(\mathbb{D}) \leqslant \nu^{(k)}(\mathbb{D})
$$

Предположим, что носители мер $\operatorname{supp} \lambda^{(k)} u \operatorname{supp} \nu^{(k)}$ лежсат в борелевских множествах $D_{k} \subset \mathbb{D} \backslash\{0\}, k=1,2, \ldots$. Положим

$$
d_{k}=\sup \left\{|z|: z \in D_{k}\right\}, \quad d_{k}^{\circ}=\inf \left\{|z|: z \in D_{k}\right\}
$$

Допустим, что удается построить последовательность полохсттельных чисел $\left\{R_{k}\right\} \operatorname{mak}$, чmo

$$
\operatorname{diam} D_{k}<R_{k}<\min \left\{1-d_{k}, d_{k}^{\circ}\right\}, \quad k=1,2, \ldots
$$

Тогда для любой меры Йенсена $\mu$ с потенциалом $V=V_{\mu}$ выполнена оценка

$$
\int V d \lambda \leqslant \sum_{k} \frac{R_{k}+\operatorname{diam} D_{k}}{R_{k}-\operatorname{diam} D_{k}}\left(\int_{D_{k}} V d \nu^{(k)}+\int_{\mathbb{D}} \int_{D_{k}} \log ^{+} \frac{R_{k}}{|z-\zeta|} d \nu^{(k)}(\zeta) d \mu(z)\right) .
$$

ДокАЗАТЕЛЬСтво. Пусть $z, z^{\prime} \in D_{k}$. Рассмотрим круг $D\left(z, R_{k}\right)$. В силу условия (3.4) с учетом определений (3.3) этот круг относительно компактен в области $\mathbb{D} \backslash\{0\}$. Тогда определено гармоническое продолжение $H_{V}$ функции Йенсена $V$ внутрь круга $D\left(z, R_{k}\right)$. Функция $H_{V}$ положительна, так как $V \geqslant 0$. При этом $z^{\prime} \in D\left(z, \operatorname{diam} D_{k}\right) \subset$ $D\left(z, R_{k}\right)$ по условию (3.4). Из субгармоничности $V$ в круге $D\left(z, R_{k}\right)$ и из неравенства Харнака [17, теорема 1.18], примененного к функции $H_{V}$ в круге $D\left(z, R_{k}\right)$, получаем

$$
\begin{aligned}
V\left(z^{\prime}\right) & \leqslant H_{V}\left(z^{\prime}\right) \leqslant \frac{R_{k}+\operatorname{diam} D_{k}}{R_{k}-\operatorname{diam} D_{k}} H_{V}(z)=\frac{R_{k}+\operatorname{diam} D_{k}}{R_{k}-\operatorname{diam} D_{k}} \cdot \frac{1}{2 \pi} \int_{0}^{2 \pi} H_{V}\left(z+R_{k} e^{i \theta}\right) d \theta \\
& =\frac{R_{k}+\operatorname{diam} D_{k}}{R_{k}-\operatorname{diam} D_{k}} \cdot \frac{1}{2 \pi} \int_{0}^{2 \pi} V\left(z+R_{k} e^{i \theta}\right) d \theta .
\end{aligned}
$$

Отсюда по условию (3.2)

$$
\begin{aligned}
\int_{D_{k}} V\left(z^{\prime}\right) d \lambda^{(k)}\left(z^{\prime}\right) & \leqslant \lambda^{(k)}\left(D_{k}\right) \sup _{z^{\prime} \in D_{k}} V\left(z^{\prime}\right) \\
& \leqslant \frac{R_{k}+\operatorname{diam} D_{k}}{R_{k}-\operatorname{diam} D_{k}} \cdot \frac{1}{2 \pi} \int_{0}^{2 \pi} V\left(z+R_{k} e^{i \theta}\right) d \theta \cdot \nu^{(k)}\left(D_{k}\right)
\end{aligned}
$$


для любой точки $z \in D_{k}$. Следовательно,

$$
\begin{aligned}
\int_{D_{k}} V\left(z^{\prime}\right) d \lambda^{(k)}\left(z^{\prime}\right) & \leqslant \frac{R_{k}+\operatorname{diam} D_{k}}{R_{k}-\operatorname{diam} D_{k}} \cdot \inf _{z \in D_{k}} \frac{1}{2 \pi} \int_{0}^{2 \pi} V\left(z+R_{k} e^{i \theta}\right) d \theta \cdot \nu^{(k)}\left(D_{k}\right) \\
& \leqslant \frac{R_{k}+\operatorname{diam} D_{k}}{R_{k}-\operatorname{diam} D_{k}} \cdot \int_{D_{k}} \frac{1}{2 \pi} \int_{0}^{2 \pi} V\left(z+R_{k} e^{i \theta}\right) d \theta d \nu^{(k)}(z) .
\end{aligned}
$$

Из классической формулы Грина для субгармонической в круге $D\left(z, R_{k}\right)$ функции $V$ имеем

$$
\frac{1}{2 \pi} \int_{0}^{2 \pi} V\left(z+R_{k} e^{i \theta}\right)=V(z)+\int_{D\left(z, R_{k}\right)} \log ^{+} \frac{R_{k}}{|z-\zeta|} d \mu,
$$

поскольку распределение масс Рисса потенциала $V=V_{\mu}$ вне нуля - это в точности мера $\mu$. Подставляя правую часть равенства (3.7) вместо внутреннего интеграла в правой части (3.6), получаем

$$
\int_{D_{k}} V\left(z^{\prime}\right) d \lambda^{(k)}\left(z^{\prime}\right) \leqslant \frac{R_{k}+\operatorname{diam} D_{k}}{R_{k}-\operatorname{diam} D_{k}} \cdot \int_{D_{k}}\left(V(z)+\int_{D\left(z, R_{k}\right)} \log ^{+} \frac{R_{k}}{|z-\zeta|} d \mu\right) d \nu^{(k)}(z) .
$$

Отсюда, применяя теорему Фубини о повторных интегралах, имеем

$$
\begin{aligned}
& \int_{D_{k}} V\left(z^{\prime}\right) d \lambda^{(k)}\left(z^{\prime}\right) \\
& \quad \leqslant \frac{R_{k}+\operatorname{diam} D_{k}}{R_{k}-\operatorname{diam} D_{k}} \cdot\left(\int_{D_{k}} V(z) d \nu^{(k)}(z)+\int_{D\left(z, R_{k}\right)} \int_{D_{k}} \log ^{+} \frac{R_{k}}{|z-\zeta|} d \nu^{(k)}(z) d \mu\right)
\end{aligned}
$$

при всех $k=1,2, \ldots$ После суммирования последних неравенств по всем $k$ в силу представлений (3.1) получаем в точности требуемое неравенство (3.5).

4. Доказательство теоремы неединственности. Следующая лемма - аналог соответствующего факта из [11, лемма 3.1], установленного в [11] для функций, мер и последовательностей в $\mathbb{C}$.

Лемма 1. Пусть $p$ - непрерывная субгармоническая функиия в $\mathbb{D}$, последовательность $\Lambda \subset \mathbb{D}$ не имеет предельных точек в $\mathbb{D} u 0 \notin \Lambda$. Допустим, ито распределение масс $\nu_{p}$ функиии р и ассочиированная с последовательностью $\Lambda$ мера $\Lambda$ представимы в виде сумм положительных мер с компактными носителями

$$
\Lambda=\sum_{k=1}^{\infty} \Lambda^{(k)}, \quad \nu_{p}=\sum_{k=1}^{\infty} \nu^{(k)}
$$

так, что для некоторой постоянной $A>0$

$$
\Lambda^{(k)}(\mathbb{D}) \leqslant A \nu_{p}^{(k)}(\mathbb{D}), \quad k=1,2, \ldots,
$$

и носители $\operatorname{supp} \Lambda^{(k)} u \operatorname{supp} \nu^{(k)}$ содержатся в борелевских множествах $D_{k} \subset \mathbb{D}$, $0 \notin D_{k}, k=1,2, \ldots$. Допустим, что выбрана последовательность $R_{k}$ такая, что

$$
\alpha \operatorname{diam} D_{k} \leqslant R_{k}<\min \left\{1-d_{k}, d_{k}^{\circ}\right\}, \quad k=1,2, \ldots,
$$


где $d_{k}, d_{k}^{\circ}$ из (3.3), а число $\alpha>1$. Положим

$$
\widehat{R}(z)=\sup \left\{R_{k}: \exists D_{k}, \operatorname{dist}\left(z, D_{k} \cap \operatorname{supp} \mu_{p}\right) \leqslant R_{k}\right\} .
$$

Если для некоторой непрерывной функции $R^{*}(z)$ на $\mathbb{D}$ выполнены неравенства

$$
\widehat{R}(z) \leqslant R^{*}(z)<1-|z|, \quad z \in \mathbb{D},
$$

то найдется голоморфная в $\mathbb{D}$ функиия $f \not \equiv 0$ такая, ито $f(\Lambda)=0$ и при некоторой постоянной $B>0$ для любой строго положительной функиии $\sigma(z)<1-|z|, z \in \mathbb{D}$, выполнена оченка

$$
\log |f(z)| \leqslant B \sup _{|z-\zeta| \leqslant \sigma(z)} \int_{0}^{2 \pi} p\left(\zeta+R^{*}(\zeta) e^{i \theta}\right) d \theta+\log \frac{1}{\sigma(z)}, \quad z \in \mathbb{D} .
$$

ДоКАЗАТЕЛЬСТво. Применим лемму сравнения с мерой $\lambda=\Lambda$ и с мерой $A \nu$ вместо меры $\nu$. Тогда неравенство (3.5) с учетом оценок (4.2), (4.3), $\alpha>1$, представления (4.1) и определения (4.4) перейдет в неравенство

$$
\begin{aligned}
\sum_{n} V_{\mu}\left(\lambda_{n}\right) & =\int V_{\mu} d \Lambda \\
& \leqslant A \sum_{k} \frac{R_{k}+R_{k} / \alpha}{R_{k}-R_{k} / \alpha}\left(\int_{D_{k}} V_{\mu} d \nu^{(k)}+\int_{\mathbb{D}} \int_{D_{k}} \log ^{+} \frac{R_{k}}{|z-\zeta|} d \nu^{(k)}(\zeta) d \mu(z)\right) \\
& \leqslant A \frac{\alpha+1}{\alpha-1}\left(\int V_{\mu} d \nu_{p}+\int_{\mathbb{D}} \sum_{k} \int_{D_{k}} \log ^{+} \frac{\widehat{R}(z)}{|z-\zeta|} d \nu^{(k)}(\zeta) d \mu(z)\right) \\
& \leqslant B^{\prime}\left(\int V_{\mu} d \nu_{p}+\iint \log ^{+} \frac{\widehat{R}(z)}{|z-\zeta|} d \nu_{p}(\zeta) d \mu(z)\right)
\end{aligned}
$$

где $B^{\prime}=A(\alpha+1) /(\alpha-1)>0$ - постоянная, справедливое для всех мер Йенсена $\mu \mathrm{c}$ потенциалом $V_{\mu}$.

Применяя обобшение (2.1) формулы Пуассона-Йенсена к первому интегралу в последней скобке из (4.7), получаем оценку

$$
\begin{aligned}
\sum_{n} V_{\mu}\left(\lambda_{n}\right) & \leqslant B^{\prime}\left(\int p(z) d \mu(z)-p(0)+\iint \log ^{+} \frac{\widehat{R}(z)}{|z-\zeta|} d \nu_{p}(\zeta) d \mu(z)\right) \\
& =B^{\prime} \int\left(p(z)+\int \log ^{+} \frac{\widehat{R}(z)}{|z-\zeta|} d \nu_{p}(\zeta)\right) d \mu(z)+C
\end{aligned}
$$

где $C=\max \left\{-B^{\prime} p(0), 1\right\}>0$ - постоянная, а неравенство (4.8) по-прежнему справедливо для всех мер Йенсена $\mu$ с потенциалом $V_{\mu}$. Из классической формулы Пуассона-Йенсена для круга $D(z, \widehat{R}(z))$ следует

$$
p(z)+\int \log ^{+} \frac{\widehat{R}(z)}{|z-\zeta|} d \nu_{p}(\zeta)=\frac{1}{2 \pi} \int_{0}^{2 \pi} p\left(z+\widehat{R}(z) e^{i \theta}\right) d \theta .
$$


Подставляя это выражение в правую часть (4.8), для всех мер Йенсена $\mu$ с потенциалом $V_{\mu}$ получаем

$$
\sum_{n} V_{\mu}\left(\lambda_{n}\right) \leqslant \int\left(B \int_{0}^{2 \pi} p\left(z+\widehat{R}(z) e^{i \theta}\right) d \theta\right) d \mu(z)+C
$$

где $B=B^{\prime} / 2 \pi>0$ и $C>0$ - постоянные. Используя (4.5), в силу монотонного возрастания средних по окружности для субгармонической функции $p$ и непрерывности $p$ из неравенства (4.9) получаем (2.4) для всех мер Йенсена $\mu$, где

$$
M(z)=B \int_{0}^{2 \pi} p\left(z+R^{*}(z) e^{i \theta}\right) d \theta
$$

- непрерьвная функция. Тогда из предложения 2 сразу следует заключение леммы 1.

Следующая лемма оценивает функцию (4.4). Пусть $a^{+} \stackrel{\text { def }}{=} \max \{a, 0\}$.

ЛЕмма 2. Пусть $R(t)$ - убывающая строго положительная функиия на $[0,1) u$ при некотором $\beta<1$ выполнено

$$
R(t) \leqslant \beta(1-t), \quad t<1,
$$

$a\left\{D_{k}\right\}$ - система подмножеств в круге $\mathbb{D}, d_{k}=\sup _{z \in D_{k}}|z|, k=1,2, \ldots$ Тогда для функиии

$$
\widehat{R}(\zeta)=\sup \left\{R\left(d_{k}\right): \exists D_{k}, \operatorname{dist}\left(\zeta, D_{k}\right) \leqslant R\left(d_{k}\right)\right\}, \quad \zeta \in \mathbb{D},
$$

в обозначении (1.3) справедлива оченка

$$
\widehat{R}(\zeta) \leqslant R\left(\frac{(|\zeta|-\beta)^{+}}{1-\beta}\right)=R_{\beta}(|\zeta|) .
$$

При этом, если $\beta \leqslant 1 / 2$ и для функиии $R(t)$ выполнено условие (1.4), то имеет место оченка

$$
t+R_{\beta}(t)<1, \quad 0 \leqslant t<1 .
$$

ДокАЗАТЕЛЬСТво. Положим $R_{k}=R\left(d_{k}\right) . \operatorname{Ecли~} \operatorname{dist}\left(\zeta, D_{k}\right) \leqslant R_{k}, \zeta \in \mathbb{D}$, то $|\zeta| \leqslant d_{k}+R_{k}$ и в силу (4.11) справедлива оценка

$$
d_{k} \geqslant|\zeta|-R_{k} \geqslant|\zeta|-\beta\left(1-d_{k}\right)=|\zeta|-\beta+\beta d_{k}
$$

Отсюда $d_{k} \geqslant(|\zeta|-\beta) /(1-\beta)$ и ввиду убьвания функции $R(t)$ получаем

$$
R\left(d_{k}\right) \leqslant R\left(\frac{(|\zeta|-\beta)^{+}}{1-\beta}\right)=R_{\beta}(|\zeta|),
$$

что и требовалось в (4.13).

Если $t<\beta \leqslant 1 / 2$, то $t+R_{\beta}(t)=t+\beta<2 \beta<1$. При $t \geqslant \beta$, но $\beta<1 / 2$ согласно (1.4а) получаем

$$
t+R_{\beta}(t) \leqslant t+\beta\left(1-\frac{t-\beta}{1-\beta}\right)=\frac{t(1-2 \beta)+\beta}{1-\beta}<1 .
$$

Наконец, при $t \geqslant \beta=1 / 2$, используя (1.4b), имеем

$$
t+R_{\beta}(t)<t+\frac{1}{2}\left(1-\frac{t-1 / 2}{1 / 2}\right)=1,
$$

и окончательно получаем (4.14). 
ДОКАЗАТЕЛЬСТВО ТЕОРЕМЫ НЕЕДИНСТВЕНноСТИ. СИстему непересекающихся борелевских множеств $\left\{D_{k}\right\}$ можно дополнить конечным набором новых борелевских множеств, предкомпактных в $\mathbb{D}$, так, что (после перенумерации) объединение множеств $D_{k}$ покрьвает $\operatorname{supp} \nu_{p}$ и по-прежнему вьполнены условия (1.7) и (1.8). Выберем в качествемер $\Lambda^{(k)}$ и $\nu^{(k)}$ сужения мер соответственно $\Lambda$ и $\nu_{p}$ на множества $D_{k}$. В частности, имеет место представление (4.1) и согласно условию (1.8) найдется постоянная $A>0$ такая, что, начиная с некоторого номера $k$, выполнены неравенства из (4.2). Пусть числа $d_{k}$ и $d_{k}^{\circ}$ определены так же, как в (3.3) и (1.7). Ввиду выполнения неравенств (4.2) при всех достаточно больших $k$ и согласно условиям (1.7) и (1.4) можно подобрать номер $k_{0}$ и число $\alpha>1$ так, что

$$
\Lambda^{(k)}(\mathbb{D}) \leqslant A \nu_{p}^{(k)}(\mathbb{D}), \quad k>k_{0}
$$

и одновременно

$$
\alpha \operatorname{diam} D_{k}<R_{k} \stackrel{\text { def }}{=} R\left(d_{k}\right) \leqslant \beta\left(1-d_{k}\right) \leqslant d_{k}^{\circ}, \quad D_{k} \subset \mathbb{D} \backslash\{0\}, \quad k>k_{0} .
$$

В частности, начиная с номера $k_{0}+1$, выполнены условия (4.3). Пусть $\nu^{0}-$ сужение меры $\nu_{p}$ на объединение множеств $\bigcup_{k=1}^{k_{0}} D_{k}, \nu^{1}=\nu_{p}-\nu^{0}$. В частности, $\nu^{0}-$ мера с компактным носителем в $\mathbb{D}$. Представим нашу функцию $p$ в виде суммы субгармонических функций $p=p_{0}+p_{1}$, где

$$
p_{0}(z)=\int_{\mathbb{D}} \log |z-\zeta| d \nu^{0}(\zeta), \quad p_{1}=p-p_{0}
$$

Представим также последовательность $\Lambda$ в виде объединения последовательностей $\Lambda=$ $\Lambda^{0} \cup \Lambda^{1}$, где $\Lambda^{0}$ - все точки последовательности $\Lambda$, попавшие в объединение $\bigcup_{k=1}^{k_{0}} D_{k}$, а последовательность $\Lambda^{1}$ состоит из всех остальньх точек.

Рассмотрим систему множеств $\left\{D_{k}^{1}\right\}, D_{k}^{1}=D_{k_{0}+1}, k=1,2, \ldots$ Согласно (4.15) и (4.16) и построению функции $p_{1}$ и последовательности $\Lambda^{1}$ выполнены условия (4.1)(4.3) леммы 1 (с системой $\left\{D_{k}^{1}\right\}$ вместо $\left\{D_{k}\right\}$, с последовательностью $\Lambda^{1}$ вместо $\Lambda$ и с функцией $p_{1}$ вместо $p$ ). В качестве функции $R^{*}$ в (4.5) по лемме 2 можно взять непрерывную функцию $R_{\beta}$ из (4.13). По лемме 1 найдется голоморфная в $\mathbb{D}$ функция $f_{1} \not \equiv 0$ такая, что $f_{1}\left(\Lambda^{1}\right)=0$ и при некоторой постоянной $B>0$ для любой строго положительной функции $\sigma(z)<1-|z|, z \in \mathbb{D}$, вьполнена оценка

$$
\log \left|f_{1}(z)\right| \leqslant B \sup _{|z-\zeta| \leqslant \sigma(z)} \int_{0}^{2 \pi} p_{1}\left(\zeta+R_{\beta}(|\zeta|) e^{i \theta}\right) d \theta+\log \frac{1}{\sigma(z)}
$$

при всех $|z|<1$.

Из представления (4.17) функции $p_{0}$ следует ее непрерывность вне носителя меры $\nu^{0}$ и, следовательно, существование некоторой постоянной $c_{0}$ такой, что для некоторого числа $t_{0}<1$

$$
B p_{0}(z) \geqslant-c_{0}, \quad t_{0} \leqslant|z|<1
$$

Далее на каждом шаге возможно увеличение числа $t_{0}<1$. 
Пусть $f_{0}$ - многочлен с последовательностью нулей $\Lambda^{0}$, являющийся, очевидно, ограниченной функцией в $\mathbb{D}$. Тогда в силу (4.19) найдется постоянная $C_{0}$ такая, что

$$
\log \left|f_{0}(z)\right| \leqslant B p_{0}(z)+C_{0}, \quad t_{0} \leqslant|z|<1, \quad f_{0}\left(\Lambda^{0}\right)=0, \quad f_{0} \not \equiv 0 .
$$

Отсюда ввиду монотонного возрастания средних по окружностям для субгармонической функции $p_{0}$ следует

$$
\log \left|f_{0}(z)\right| \leqslant B \sup _{|z-\zeta| \leqslant \sigma(z)} \int_{0}^{2 \pi} p_{0}\left(\zeta+R_{\beta}(|\zeta|) e^{i \theta}\right) d \theta+C_{0}, \quad t_{0} \leqslant|z|<1
$$

для любой строго положительной функции $\sigma(z)<1-|z|, z \in \mathbb{D}$.

Напомним, что $\Lambda=\Lambda^{0} \cup \Lambda^{1}, p=p_{0}+p_{1}$. Следовательно, объединяя последнюю оценку с (4.18), для голоморфной в $\mathbb{D}$ функции $f=f_{0} f_{1} \not \equiv 0, f(\Lambda)=0$, получаем

$$
\log |f(z)| \leqslant B \sup _{|z-\zeta| \leqslant \sigma(z)} \int_{0}^{2 \pi} p\left(\zeta+R_{\beta}(|\zeta|) e^{i \theta}\right) d \theta+\log \frac{1}{\sigma(z)}+C_{0}
$$

при $t_{0} \leqslant|z|<1$ для любой строго положительной функции $\sigma(z)<1-|z|, z \in \mathbb{D}$. Отсюда по условию $(\mathbf{R})$ следует существование функции $p_{1} \in \mathscr{P}$ и постоянных $C_{1}^{\prime}, C_{2}^{\prime}>0$ таких, что

$$
\log |f(z)| \leqslant C_{1}^{\prime}\left(\sup _{|z-\zeta| \leqslant \sigma(z)} p_{1}(\zeta)+\frac{1}{C_{1}^{\prime}} \log \frac{1}{\sigma(z)}\right)+C_{2}^{\prime}, \quad t_{0} \leqslant|z|<1
$$

для любой строго положительной функции $\sigma(z)<1-|z|, z \in \mathbb{D}$. Выберем в качестве функции $\sigma(z)$ соответствие, которое каждой точке $z$ сопоставляет число $\sigma$ в условии $(\mathbf{S})$ при $C=1 / C_{1}^{\prime}$. Тогда согласно последней оценке по условию $(\mathbf{S})$ найдутся постоянные $C_{1}, C_{2}>0$ такие, что

$$
\log |f(z)| \leqslant C_{1} p_{2}(z)+C_{2}, \quad t_{0} \leqslant|z|<1
$$

для некоторой функции $p_{2} \in \mathscr{P}$. Учитьвая непрерывность функции $p_{2}$, увеличив, если необходимо, постоянную $C_{2}$, оценку (4.20) можно продолжить на все $z \in \mathbb{D}$. Таким образом, $f \in A \mathscr{P}$. Вместе со свойствами $f \not \equiv 0$ и $f(\Lambda)=0$ это означает, что $\Lambda$ последовательность неединственности для $A \mathscr{P}$, и теорема неединственности доказана. 


\section{СПИСОК ЦИТИРОВАННОЙ ЛИТЕРАТУРЫ}

[1] Шведенко С. В. Классы Харди и связанные с ними пространства аналитических функций в круге, поликруге и шаре // Итоги науки и техники. Сер. матем. анализ. 1985. Т. 23. С. 3-124.

[2] Djrbashian A., Shamoyan F. A. Thopics in the theory of $A_{\alpha}^{p}$ spaces. Leipzig: Teubner-Texte, 1988.

[3] Шамоян Ф. А. Факторизационная теорема М. М. Джрбашяна и характеристика нулей аналитических в круге функций с мажорантой конечного роста // Изв. АН АрмССР. Матем. 1978. T. 13. № 5-6. С. 405-422.

[4] Шамоян Ф. А. О нулях аналитических в круге функций, растущих вблизи границы // Изв. АН АрмССР. Матем. 1983. Т. 18. №1. С. 15-27.

[5] Horovitz C. Zero sets and radial zero sets in function spaces // J. Anal. Math. 1995. V. 65. P. $145-159$.

[6] Korenblum B. An extension of the Nevanlinna theory // Acta Math. 1975. V. 135. № 3-4. P. 187-219.

[7] Beller E. Factorization for non-Nevanlinna classes of analytic functions // Israel J. Math. 1977. V. 27. № 3-4. P. 320-330.

[8] Beller E., Horovitz C. Zero sets and random zero sets in certain function spaces // J. Anal. Math. 1994. V. 64. P. 203-217.

[9] Bruna J., Massaneda X. Zero sets of holomorphic functions in the unit ball with slow growth // J. Anal. Math. 1995. V. 66. P. 217-252.

[10] Чередникова Л. Ю., Хабибуллин Б.Н. Множества неединственности для весовых алгебр голоморфных в круге функций // Труды международной конференции "Комплексный анализ, дифференциальные уравнения и смежные вопросы”. І. Комплексньй анализ. Уфа, 2000. C. $195-200$.

[11] Хабибуллин Б.Н. Неконструктивные доказательства теоремы Берлинга-Мальявена о радиусе полноты и теоремы неединственности для целых функций // Изв. РАН. Сер. матем. 1994. T. 58. № 4. C. 125-148.

[12] Koosis P. Leçons sur le théorème de Beurling et Malliavin. Montréal: Les Publications CRM, 1996.

[13] Хабибуллин Б. Н. Множества единственности в пространствах целых функций одной переменной // Изв. АН СССР. Сер. матем. 1991. Т. 55. № 5. С. 1101-1123.

[14] Хабибуллин Б. Н. Теорема о наименьшей мажоранте и ее применения. І. Целые и мероморффные функции // Изв. РАН. Сер. матем. 1993. Т. 57. № 1. С. 129-146.

[15] Khabibullin B. N. Dual approach to certain questions for weighted spaces of hol-omorphic functions // Israel Mathematical Conference Proceedings (Tel-Aviv, 1997). V. 15. Tel-Aviv, 1997. P. 207-219.

[16] Хабибуллин Б.Н. Двойственное представление суперлинейных функционалов и его применения в теории функций. II // Изв. РАН. Сер. матем. 2001. Т. 65. № 5. С. 167-190.

[17] Хейман У., Кеннеди П. Субгармонические функции. М.: Мир, 1980. 